\title{
The digitized first Byurakan survey - DFBS
}

\author{
A. M. Mickaelian ${ }^{1}$, R. Nesci ${ }^{2}$, C. Rossi ${ }^{2}$, D. Weedman ${ }^{3}$, G. Cirimele ${ }^{4}$, L. A. Sargsyan ${ }^{1}$, \\ L. K. Erastova ${ }^{1}$, K. S. Gigoyan ${ }^{1}$, G. A. Mikayelyan ${ }^{5}$, E. Massaro ${ }^{2}$, S. Gaudenzi ${ }^{2}$, \\ J. Houck ${ }^{3}$, D. Barry ${ }^{3}$, L. D'Amante ${ }^{4}$, and P. Germano ${ }^{4}$ \\ 1 Byurakan Astrophysical Observatory and Isaac Newton Institute of Chile, Armenian branch, Byurakan 378433, \\ Aragatzotn province, Armenia \\ e-mail: aregmick@apaven.am \\ 2 Università di Roma "La Sapienza", Piazzale A. Moro 2, 00185 Roma, Italy \\ e-mail: Roberto.Nesci@uniroma1.it \\ 3 Cornell University, 220 Space Sciences Building, Ithaca, NY 14853-6801, USA \\ e-mail: dweedman@isc.astro.cornell.edu \\ 4 MIGG s.r.l., via Pertini 61, 87030 Rende, Italy \\ e-mail: cirimele@migginformatica.com \\ 5 Yerevan State University, Alex Manoogian str. 1, Yerevan 375049, Armenia
}

Received 12 August 2006 / Accepted 8 January 2007

ABSTRACT

\begin{abstract}
Context. The First Byurakan Survey (FBS), also known as the Markarian Survey, is the largest low resolution spectroscopic survey of the sky and led to the discovery of 1500 UV-excess (UVX) galaxies and starburst galaxies. The FBS plates have also been used to search for UVX stellar objects, late-type stars, and for the identification of unusual infrared sources.

Aims. The Digitized First Byurakan Survey (DFBS) provides the astronomical community with a digitized version of the FBS images and with the extracted spectra for the objects present in the plates.

Methods. Nearly 2000 plates have been scanned and stored and programs were developed to compute the astrometric solution, extract the spectra, and apply wavelength and photometric calibration for the objects present in the plates.

Results. The DFBS database and catalog of objects has been assembled. The DFBS database contains data for 20000000 objects present in the survey and provides tools for accessing the DFBS.

Conclusions. New scientific projects as well as existing surveys will benefit by the digitized images and the ready-to-use extracted spectra which will allow an efficient computer-based analysis of the dataset.
\end{abstract}

Key words. surveys - techniques: spectroscopic - methods: data analysis

\section{The FBS and previous results}

The First Byurakan Survey (FBS) was the first systematic objective prism survey of the extragalactic sky. It was conducted by Markarian, Lipovetski and Stepanian in 1965-1980 at the Byurakan Astrophysical Observatory with the $1 \mathrm{~m}$ Schmidt telescope and $1.5^{\circ}$ prism. At present, the FBS is the largest area spectral survey, covering $17000 \mathrm{deg}^{2}$ of all the northern sky and part of the southern sky at high galactic latitudes, with a total of about 20000000 objects in the entire survey (Markarian et al. 1989).

The original aim was the search for galaxies with UV excess (Mazzarella \& Balzano 1986; Markarian et al. 1989, 1997 - catalogue No. VII/172 at CDS). Studies of the Markarian galaxies early in the survey led to the spectral classification of Seyfert Galaxies (Weedman \& Khachikian 1971), and to the first definition of starburst galaxies (Weedman 1977).

The huge amount of spectral information contained in the plates allowed the development of several other projects based on the FBS, the most important being the discovery and investigation of blue stellar objects (Abrahamian \& Mickaelian 1996; Mickaelian et al. 2001, 2002, CDS catalogue No. II/223); a survey for late-type stars (Gigoyan et al. 2002) and the optical identifications of sources from the IRAS catalog (IRAS 1988).
The sample of stellar objects is available at CDS (ByurakanIRAS Stars - BIS, catalogue No. III/237, Mickaelian \& Gigoyan 2001), and a similar catalogue for IRAS galaxies (ByurakanIRAS Galaxies - BIG, Mickaelian \& Sargsyan 2004) will soon appear at CDS.

All of these results were obtained by eye inspection of the plates at the Byurakan Observatory using a lens. The number and classes of new objects discovered within the FBS made clear the need for open access to this information by the entire astronomical community.

\section{The Digitized First Byurakan Survey (DFBS)}

Until 2003, the FBS plate database was not accessible to the astronomical community. Data for many plates were missing, and the plate numbers were coded such that there was no easy access to any definite field, even with access to the original plates at Byurakan Observatory. We have now identified the plate fields, compiled a complete list of the FBS plates with all necessary data, and made this catalog available since February 2003 through the Wide-Field Plate Database (WFPDB) home page at http://www. skyarchive.org/data/ (Mickaelian et al. 2005; catalogue No. VI/116 at CDS). 
In 2002, we began digitizing the entire plate set, providing the astrometric plate solution, and developing an automatic procedure to extract the spectra contained in the plates in order to allow a computer-based reanalysis of the data set. The work that led to the final Digitized First Byurakan Survey (DFBS) was carried out as a collaboration between the Byurakan Astrophysical Observatory (Armenia), the University of Roma "La Sapienza" (Italy), and Cornell University (USA).

In the following subsections, we briefly describe the steps to build the DFBS and user interface, the current state of the project, and future plans. A detailed description of the reduction procedures is available in the web page, which has been created to allow access to the DFBS database by the astronomical community, as described below.

\subsection{Scanning}

Digitizing of the FBS plates started in 2002. After several test scans on a set of plates, all of the FBS plates were digitized with an Epson Expression 1680 Pro scanner at the Byurakan observatory. The scanning resolution was $1600 \mathrm{dpi}$ so the pixel size is about 16 microns. The typical length of an FBS spectrum is $\sim 1.7 \mathrm{~mm}$, which gives 107 pixels along the wavelength direction.

An "ad hoc" program SCANFITS written by Stefano Mottola (DLR - Institute of Planetary Research, Berlin) allows the resulting image to be written directly in FITS format with corresponding information about the plate in the header. All 1874 plates in 1139 fields were digitized and stored on DVDs.

\subsection{Astrometric solution}

The red cutoff of the FBS spectra, defined as the point where the intensity is half of the peak value, is relatively sharp and can be used as a reference point for position (as well as for wavelength calibration; see Sect. 2.4), but it is mildly sensitive to the brightness and spectral type (color) of the object. The accuracy of definition for this cutoff is $\sim 0.2^{\prime \prime}$. The software for the astrometric solution was written by Hagen by adapting the dedicated software for the Hamburg Quasar Survey (Hagen et al. 1995). The Second Guide Star Catalogue (GSC-2) was used as the reference positional input. Beginning with the plate center and the brightest stars in the field, an iterative procedure converges to the astrometric solution. Up to 800 stars of progressively decreasing brightness were used in each field, and up to 7 approximations were carried out to achieve the best solution. The plate scale is $1.54^{\prime \prime} /$ pixel. The best accuracy achieved was $0.87^{\prime \prime} \mathrm{rms}$, and the typical accuracy, $\sim 1^{\prime \prime}$ rms or 0.6 pixel, is sufficient for confident object identification (a spectrum is typically 5 pixels wide). The total time needed for the astrometric solution of each plate was $\sim 5 \mathrm{~min}$, and the procedure was applied for all 1874 DFBS plates.

A few examples from the scanned FBS plates are illustrated in Fig. 1. In these images, the signal sharply drops to zero at long wavelengths (top of the spectra in Fig. 1) owing to the sensitivity cutoff of the emulsion in the red.

\subsection{Density calibration and spectral extraction}

Dedicated software named bSpec was developed to provide automatic extraction and classification of the spectral data in a DFBS image. This software was developed by the MIGG s.r.l. team as a collaborative project with the "La Sapienza"

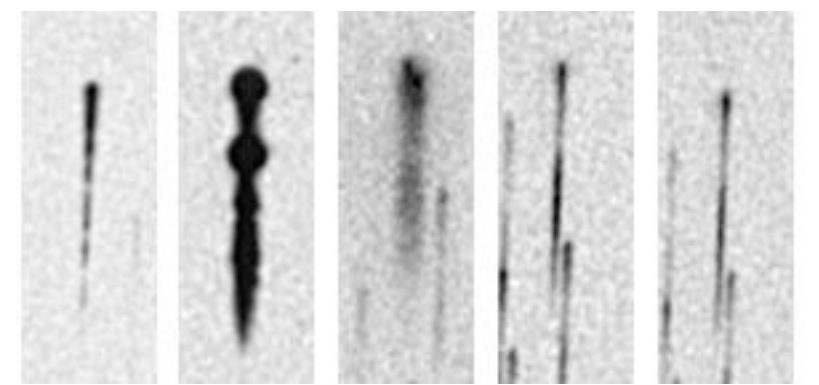

Fig. 1. Examples of two dimensional spectra present in DFBS images; objects from left to right are: the carbon star FBS 0644+616, the planetary nebula NGC 6210, the pair of galaxies Mrk 266, and the cataclismic variable DQ Her in two different photometric states.

University group; it was coded under Linux using the Borland Kylix compiler.

The FBS plates do not have photometric calibration, and it is not possible to build a characteristic curve for each plate. Therefore, we converted the original Data Numbers $(D N)$ output of the scanning to relative intensity according to the formula (De Vaucouleurs 1968): $I=(V-B) /(T-B)$, where $I$ is the intensity (in arbitrary units), $V$ is the average $D N$ value for the unexposed plate, $B$ is the average for the darkest pixels, and $T$ is the data number for a given pixel. In the immediate future, we will begin developing an absolute calibration using the energy distribution of known stars within the plates.

The extraction of individual spectra from the two dimensional images was performed using several steps. To define the object position and spectral contours, we downloaded a list of objects with $B \leq 17$ from the USNO catalogue. Starting from the catalogue coordinates, each spectrum was recentered using a combination of the two parameters peak position (the pixel of maximum density in the spectrum) and baricenter (the center of the two-dimensional fit to the spectrum). For each image, a mean spectrum direction angle was computed using the distribution of the entire set of catalogue objects and then adopted as the spectrum direction for all spectra on the plate.

All spectra of the objects from the catalogue were then extracted automatically, corrected for the adjacent sky. The abscissa of each spectrum was set to have pixel 20 at the "red head", where the sensitivity of the plates sharply drops to zero. A disadvantage of this approach is that we lose variable or moving objects present in the plates but fainter than the adopted threshold in the USNO catalogue (or absent in the catalogue).

Therefore, we have tested a second procedure for extraction of the spectra based on the software SExtractor (Bertin \& Arnouts 1996). We found this method to be good for finding all objects, although defects and artifacts are taken as objects and some faint objects are missed. We concluded that this procedure can be used for relatively low-density fields and brighter objects (e.g., for overlapped images, always the brightest spectra is taken). In the future, it may be applied to produce a database somewhat modified from the present one.

\subsection{Wavelength calibration and multiband photometry}

To determine the wavelength calibration, we used some wellexposed spectra of planetary nebulae, white dwarfs, subdwarfs, cataclysmic variables, and QSOs, which have broad Balmer, Helium, and other lines. We obtained only an approximate wavelength calibration scale because the dispersion is strongly nonlinear, from about $22 \AA /$ pixel at the blue edge of a spectrum 


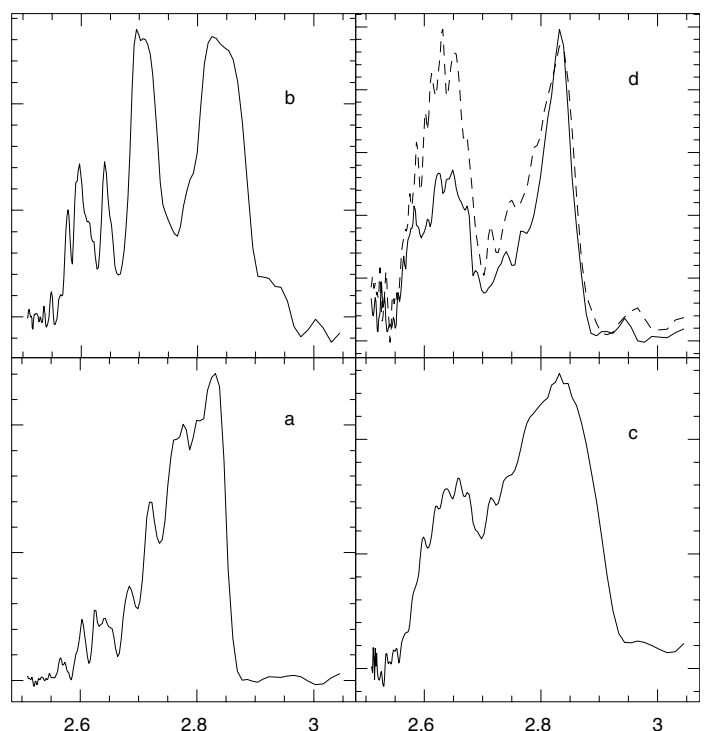

Fig. 2. Extracted spectra of the objects shown in Fig. 1: a) FBS 0644+616; b) NGC 6210; c) MRK 266; d) DQ Her (in two different photometric states). The scale of the $x$ axis is $\log (\lambda)$ with wavelength in $\mathrm{nm}$, the intensities are in arbitrary units.

to about $60 \AA$ /pixel at the red edge, with mean dispersion $32.7 \AA /$ pixel and dispersion of about $28.5 \AA /$ pixel at $\mathrm{H} \gamma$. The one-dimensional spectra of the objects shown in Fig. 1 are shown in Fig. 2 as they are stored in the database.

Photometric information from the DFBS spectra can be obtained by integrating the spectra blueward and redward of the green sensitivity gap typical of IIF plates, thereby deriving an instrumental "blue" and "red" magnitude; these spectral regions

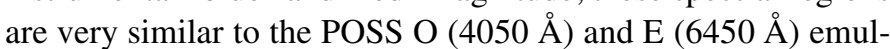
sion sensitivity ranges and can be reliably related, therefore, to the $B$ and $R$ magnitudes given in the USNO catalogue. A magnitude calibration for each plate was derived from the stars contained in an area of $4000 \times 4000$ pixels in the central part of the plate. Instrumental $B$ and $R$ magnitudes were evaluated by integrating the spectrum between pixels 20-40 $(R)$ and 55-90 $(B)$. A polynomial fit of these instrumental magnitudes compared to the USNO magnitudes provided a calibration curve which was then used to compute the DFBS magnitudes for all objects in the plates. Objects brighter than $B=11$ are generally overexposed in the DFBS so their magnitudes are not very accurate, nor are the faint objects having magnitudes near the plate limit. To illustrate the internal consistency of the calibration process for the FBS plates, we show in Fig. 3 a comparison of data from two plates in the same field. For both $B$ and $R$, the typical agreement is $\sim 0.5^{\mathrm{m}}$ in the range $13^{\mathrm{m}}-16^{\mathrm{m}}$.

\section{The DFBS database and the web page}

All spectra extracted with $b S$ pec were assembled in a database which contains for each object the one-dimensional spectrum plus additional information including an identification label in the coordinates hhmmss+ddmmss, the coordinates RA, Dec at equinox 2000, $B$ and $R$ magnitudes from the extracted spectra obtained as described above, the local background value, and a quality flag computed on the basis of the brightness of the object and the possible blending with other stars.

The DFBS database containing the extracted spectra is presently stored on a dedicated PC at the "La Sapienza"

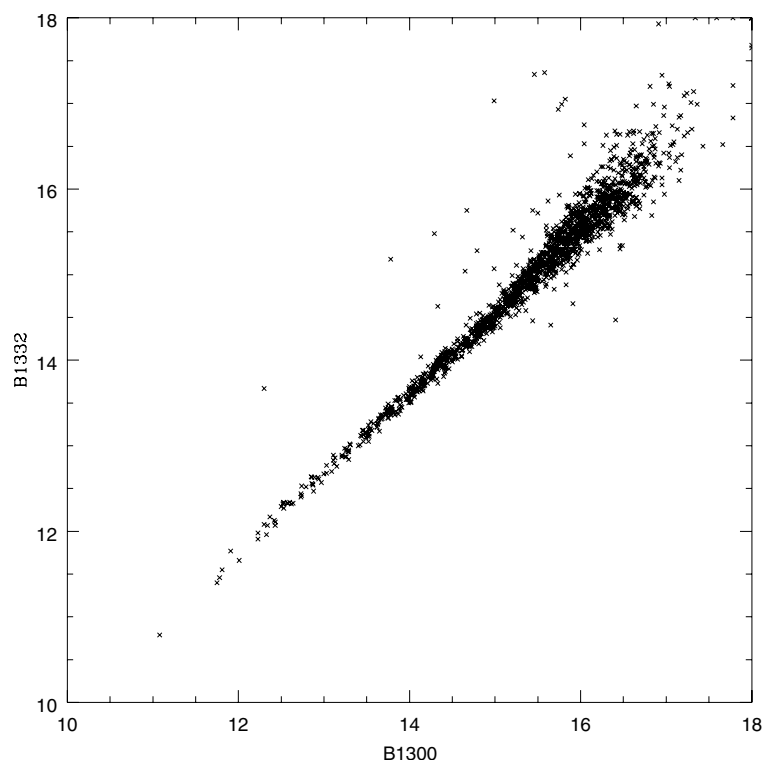

Fig. 3. Photometric agreement between two DFBS plates taken for the same field in $B$ band $(\lambda=4500 \AA)$. Both DFBS plates contain objects up to $17.5^{\mathrm{m}}$; rms is 0.12 for the $B$ and 0.09 for the $R$ instrumental magnitudes.

University in Rome and can be accessed through the web interface http://byurakan.phys.uniroma1.it/.

The user interface has a menu type structure. Several palettes give general information on the FBS, the main characteristics of the observational material, and the main results obtained from the plates. In particular, the keys sky-coverage and platelist contain detailed information on individual plates, such as type of emulsion, observation date, observer, and accurate central coordinates from the plate center computed after application of the astrometric solution.

The digitization and reduction procedures are also described in detail. In the explore menu portion, the images are visualized and can be compared with the corresponding direct plates from the Digital Sky Survey; the extracted spectra of individual objects can also be displayed and stored to be downloaded later. Users cannot run the bSpec software on a remote machine to create a personal database, but they may download portions of the plates in FITS format and perform their own analysis of the images.

\section{Future developments}

The electronically-accessible DFBS is the primary result of our project. Use of the digitized spectra will increase the efficiency of object selection for various scientific goals. For example, inspection of low dispersion spectra is often the most efficient way for initially checking the targets to identify optical sources discovered in X-ray, IR, and radio surveys.

The next step of our work will consist of the development of criteria for automatic spectral classification. Different methods are currently being tested and will be included in a future version of the database. One approach is based on developing template spectra for different types of objects using available catalogues and averaging the DFBS spectra for each type. A second approach is based on a numerical classification scheme which utilizes multivariate relations such as relations between magnitudes and widths of the spectra (for separation of stellar and diffuse objects), spectral energy distributions, ratio of the red/blue 
part of the spectra, length of the spectra, etc. Such classifications derive from criteria developed during the selection of blue stellar objects, red stars, and identification of IRAS sources.

In the Introduction, we briefly mentioned some of the previous surveys based on the FBS plates. The digitized images of the DFBS and the resulting template spectra will allow faster, more quantitative identification of targets. We are confident that these new tools will not only benefit previously initiated projects, but that new research ideas will also originate from the Digitized First Byurakan Survey.

Acknowledgements. We acknowledge grants from ANSEF in 2002 and 2007, USA NSF in 2003-2004, REGIONE CALABRIA and U.E. (FESR) in 2005-2006, and CRDF in 2007.

The University "La Sapienza" partially supported the project with special funds for International collaborations.

The authors are very thankful to Dr. Hans Hagen (Hamburg Observatory, Germany) for his astrometric solution software modified and kindly provided for the DFBS plates.

\section{References}

Abrahamian, H. V., \& Mickaelian, A. M. 1996, Ap, 39, 531

Bertin, E., \& Arnouts, S. 1996, A\&AS, 117, 393

De Vaucouleurs, G. 1968, Applied Optics, 7, 1513
Gigoyan, K. S., Abrahamian, H. V., Azzopardi, M., \& Russeil, D. 2002, Ap, 45, 322

GSC2.2 2001, The Guide Star Catalogue, Version 2.2.01 Space Telescope Science Institute (STScI) and Osservatorio Astronomico di Torino

Hagen, H.-J., Groote, D., Engels, D., \& Reimers, D. 1995, A\&AS, 111, 195

IRAS 1988, The Point Source Catalog. Joint IRAS Science Working Group, NASA, Washington, DC

Markarian, B. E., Lipovetski, V. A., Stepanian, J. A., et al. 1989, Commun. Special Astrophys. Obs., 62, 5

Markarian, B. E., Lipovetski, V. A., \& Stepanian, J. A. 1997, First Byurakan Survey (FBS), CDS, Strasbourg; http://vizier.u-strasbg.fr/ viz-bin/VizieR?-source=VII/172/fbs

Mazzarella, J. M., \& Balzano, V. 1986, ApJS, 62, 751

McMahon, R. G., Irwin, M. J., \& Maddox, S. J. 2000, The APM-North Catalogue, Institute of Astronomy, Cambridge, CB3 OHA, UK

Mickaelian, A. M., \& Gigoyan, K. S. 2001, Ap, 44, 222

Mickaelian, A. M., \& Sargsyan, L. A. 2004, Ap, 47, 251

Mickaelian, A. M., Gonçalves, A. C., Véron-Cetty, M. P., \& Véron, P. 2001, Ap, 44,14

Mickaelian, A. M., Balayan, S. K., Ilovaisky, S. A., et al. 2002, A\&A, 381, 894

Mickaelian, A. M., Hagen, H.-J., Sargsyan, L. A., \& Mikayelyan, G. A. 2005, Digitized First Byurakan Survey plate database, CDS, Catalogue No. VI/116, Strasbourg

Monet, D., Bird, A., Canzian, B., et al. 1996, The USNO-A2.0 Catalogue, Washington DC: US Naval Observatory

Monet, D. G., Levine, S. E., Casian, B., et al. 2003, AJ 125, 984

Weedman, D. W. 1977, Vistas Astron., 21, 55

Weedman, D. W., \& Khachikian, E. Ye. 1971, Ap, 7, 389 